\title{
Enhancing Executive Functions Through Social Interactions: Causal Evidence Using a Cross-Species Model
}

\author{
Rosemarie E. Perry ${ }^{1 *}$, Stephen H. Braren1, Millie Rincón-Cortés ${ }^{2,3}$, \\ Annie N. Brandes-Aitken1, Divija Chopra1, Maya Opendak,3, Cristina M. Alberini", \\ Regina M. Sullivan ${ }^{2,3}$ and Clancy Blair ${ }^{1,5 *}$
}

\begin{abstract}
${ }^{1}$ Department of Applied Psychology, New York University, New York, NY, United States, ${ }^{2}$ Emotional Brain Institute, Nathan Kline Institute for Psychiatric Research, Orangeburg, NY, United States, ${ }^{3}$ Department of Child and Adolescent Psychiatry, New York University School of Medicine, New York, NY, United States, ${ }^{4}$ Center for Neural Science, New York University, New York, NY, United States, ${ }^{5}$ Department of Population Health, New York University School of Medicine, New York, NY, United States
\end{abstract}

OPEN ACCESS

Edited by:

Gian Marco Marzocchi,

University of Milano-Bicocca, Italy

Reviewed by:

Eric Pakulak,

University of Oregon, United States

Vrinda Kalia,

Miami University, United States

*Correspondence:

Rosemarie E. Perry

rosemarie.perry@nyu.edu

Clancy Blair

clancy.blair@nyu.edu

Specialty section:

This article was submitted to Developmental Psychology,

a section of the journal

Frontiers in Psychology

Received: 24 June 2019 Accepted: 21 October 2019 Published: 19 November 2019

Citation:

Perry RE, Braren $\mathrm{SH}$,

Rincón-Cortés M, Brandes-Aitken AN, Chopra D, Opendak M, Alberini CM, Sullivan RM and Blair C (2019) Enhancing Executive Functions Through Social Interactions: Causal Evidence Using a Cross-Species Model. Front. Psychol. 10:2472. doi: 10.3389/fpsyg.2019.02472
It has long been theorized that humans develop higher mental functions, such as executive functions (EFs), within the context of interpersonal interactions and social relationships. Various components of social interactions, such as interpersonal communication, perspective taking, and conforming/adhering to social rules, may create important (and perhaps even necessary) opportunities for the acquisition and continued practice of EF skills. Furthermore, positive and stable relationships facilitate the development and maintenance of EFs across the lifespan. However, experimental studies investigating the extent to which social experiences contribute causally to the development of EFs are lacking. Here, we present experimental evidence that social experiences and the acquisition of social skills influence the development of EFs. Specifically, using a rat model, we demonstrate that following exposure to early-life adversity, a socialization intervention causally improves working memory in periadolescence. Our findings combined with the broader literature promote the importance of cultivating social skills in support of EF development and maintenance across the lifespan. Additionally, cross-species research will provide insight into causal mechanisms by which social experiences influence cognitive development and contribute to the development of biologically sensitive interventions.

Keywords: executive function, social competence, early-life adversity, poverty, social skills, social behavior, development, longitudinal

\section{INTRODUCTION}

The cognitive control abilities that enable holding and manipulating information in mind, the flexible shifting of attention between tasks, and inhibiting impulses and responses to stimuli are critical thinking skills that assist reasoning, planning, self-regulation, and management of one's life. These higher-order cognitive abilities-called executive functions (EFs)-develop across the lifespan and are enhanced or diminished by a variety of experiential factors, especially early in life, 
such as environmental stimulation or stress/adversity (Perry et al., 2018b), and even physical fitness (e.g., body mass index, physical exercise) (Verburgh et al., 2014; Blair et al., 2019). Prior studies have demonstrated that these experiential factors can be successfully leveraged as points of intervention, with EF skills improving following interventions promoting stress reduction (Zelazo and Lyons, 2012) or physical exercise (Verburgh et al., 2014). However, a lesser acknowledged factor by which EF skills may also be promoted is through interpersonal experiences. This is despite strong evidence from developmental research and longstanding theory that cognitive development occurs within the context of positive social interactions and relationships (Vygotsky, 1978; Carlson, 2009; Lewis and Carpendale, 2009; Moriguchi, 2014; van Lier and Deater-Deckard, 2016).

While EF and social development are traditionally considered to be distinct domains of development, they are increasingly understood to be functionally connected. The majority of research regarding the social origins of EFs has focused on caregiver scaffolding of EF development through social interactions with their infants (e.g., Landry et al., 2002; Bibok et al., 2009; Hughes and Ensor, 2009; Roskam et al., 2014). This large body of research provides strong support that sensitive caregiving facilitates EF development. Furthermore, increasing evidence suggests that social processes influence EF development not only in infancy, but also across later development as peers become more central in youth's lives. For example, in preschool, engaging in pretend play with peers is associated with improved self-regulation (Lindsey and Colwell, 2003). Playful interactions with peers are also associated with EF development, including cognitive flexibility (Bateson, 2005) and inhibitory control (Peterson and Flanders, 2005). Even in adolescence and adulthood, peer problems, such as peer victimization, rejection, and social exclusion, have been associated with impaired EF skills (Baumeister et al., 2002, 2005; Holmes et al., 2016). Despite these findings, the extent to which social interactions with peers may function as causal mechanisms supporting EFs is not understood.

The attainment of appropriate social skills through social interactions with peers (in addition to caregivers) may be an important driving component of EF development. In line with this idea, we recently reported findings of a novel developmental pathway whereby social competence through EF longitudinally mediated the impact of cumulative poverty-related adversities on academic achievement across the early school years (Perry et al., 2018a). Specifically, social competence in Kindergarten through EFs at Grade 1 longitudinally mediated a negative association between early-life poverty-related cumulative risk exposure and academic skills at Grade 2. These findings are in line with a growing literature that suggests that the development of social competence may be functionally linked with the development of EFs (e.g., Riggs et al., 2006; Carlson, 2009; Lewis and Carpendale, 2009; Moriguchi, 2014; van Lier and Deater-Deckard, 2016). Additionally, these results indicate that social competence may be a key mechanism by which early-life adversity impacts EF development.

Taken together, our findings paired with a broader body of literature and longstanding theory suggest that higherorder cognitive development might be facilitated, at least in part, by targeting the improvement of social skills and social interactions with caregivers and peers. Moreover, this developmental relation may be especially important for children reared in adverse environments. Indeed, a few randomized controlled trials (RCTs) provide further support for this idea. For example, interventional school curricula, such as Tools of the Mind, have incorporated Vygotskian principles into their design by not only directly scaffolding EF development, but also incorporating social pretend play to positively impact EF development (Bodrova and Leong, 1996; Diamond et al., 2007; Blair and Raver, 2014; Sasser et al., 2017). However, looking beyond the earliest school years, there is a paucity of RCTs experimentally testing if interventions that target social processes positively impact EF development. This gap in the literature persists despite well-established evidence that $\mathrm{EF}$ development is protracted and remains amenable to experiential input well into adolescence and adulthood (Perry et al., 2018b). Furthermore, the field is currently limited in its understanding of the causal mechanisms by which social processes operate to influence EF development, which would ultimately inform design and implementation (e.g., developmental timing) aspects of interventions to maximize effect sizes.

These gaps in the literature are likely due to normal limitations that human developmental researchers face. While prior research, including our own, has benefited from longitudinal data to begin to understand how social processes influence EF development, most studies are based on non-experimental, correlational data which limits our inferences regarding causal relations. Furthermore, we face difficulties in readily discerning causeeffect relations between social processes and EF development due to lack of experimental control within research designs involving humans. Thus, in the present study, we expanded upon our prior human findings (Perry et al., 2018a) by leveraging a rodent model with high internal validity to experimentally test our overarching hypothesis that EF development can be enhanced by targeting the improvement of social skills through facilitated social interactions. We focus specifically on the functional interplay between social development and working memory, a core $\mathrm{EF}$ which involves the ability to hold in mind, manipulate, and update information in one's memory (Diamond, 2013). Working memory can be readily assessed in rodent models by using a widely used spontaneous alternation task, which is based on the tendency of rodents to explore a prior unexplored arm of a maze, and thus requires that the rodent remember which maze arms were most frequently visited (e.g., Lalonde, 2002; Hughes, 2004; Liet et al., 2015; Kraeuter et al., 2019). Importantly, spontaneous alternation also occurs in humans and has been demonstrated as early as 18 months of age (Vecera et al., 1991). Working memory is an important component of social competence as it is essential for organizing, inhibiting, and executing behavior (Riggs et al., 2006). Indeed, working memory has been associated with the facilitation of social development (Riggs et al., 2006). Furthermore, working memory develops into young adulthood and remains malleable (especially in childhood), such that working memory skills can be influenced by training (Klingberg et al., 2002, 2005) and social experiences (Perry et al., 2018b). 
Thus, we employed a rodent model of early-life scarcityadversity, which induces atypical mother-infant interactions (Perry et al., 2019) and altered social behavior across development (Raineki et al., 2012, 2015; Rincón-Cortés and Sullivan, 2016), to experimentally test if a peer socialization intervention could improve working memory in peri-adolescence. Based upon Vygotskian theory linking cognitive development to social processes, as well as prior findings that our rodent model of early-life scarcity-adversity causes social behavior problems in later life, we hypothesized that scarcity-adversity rearing would also produce cognitive development problems, as assessed via working memory performance in peri-adolescence. We additionally sought to replicate and expand upon previous results demonstrating that early-life scarcity-adversity would cause social behavior problems in juvenile and adolescent rats (Raineki et al., 2012, 2015; Rincón-Cortés and Sullivan, 2016). Furthermore, drawing from our prior human research findings suggesting that social development influences EF development (Perry et al., 2018a), we hypothesized that socializing a scarcityadversity reared subject with a control reared rat (via co-housing) would improve the scarcity-adversity reared subject's social behavior and working memory performance. We tested this using a peer socialization intervention spanning from time of weaning until time of testing in peri-adolescence, a developmental period which encompasses the maturation of social behavior and is increasingly thought of as a period in which neurodevelopment is sensitive to social experiences (Sisk and Foster, 2004; Schulz et al., 2009; Wei et al., 2011; Fuhrmann et al., 2015). While this rodent model is not meant to supersede the need for future human RCTs examining the efficacy of peer socialization interventions for the improvement of EFs, it serves as a valuable tool with which we can efficiently test our research questions using a tightly controlled experimental design. Furthermore, our rodent model welcomes future experiments for the assessment of specific behavioral and neurobiological mechanisms by which social interactions influence cognitive development, which would provide valuable insight into the design of mechanism-based, developmentally sensitive, biologically informed interventions.

\section{MATERIALS AND METHODS}

\section{Subjects}

Male and female Long Evans rats were bred and raised in a temperature $\left(20 \pm 1^{\circ} \mathrm{C}\right)$ - and light (12-h light/dark cycle)controlled room in an animal facility to provide a controlled rearing environment for all subjects. Subjects were born on postnatal day (PN) 0 and culled to 12 pups (six males, six females) on PN1. With the exception of our scarcity-adversity reared subjects (described in methods below), animals were housed with their mother in polypropylene cages $(34 \times 29 \times 17 \mathrm{~cm})$ with ad libitum food (Purina LabDiet \#5001) and water, as well as ample wood shavings materials for nest building. Animals were weaned from their mother at PN23 and housed with one age- and sex-matched cage mate in a polypropylene cage $(34 \times 29 \times 17 \mathrm{~cm})$ with access to ample wood shavings and ad libitum food (Purina LabDiet \#5001) and water. Animals were tested once in peri-adolescence (PN37-47, the time immediately prior to and during the onset of puberty) and each subject was only used once, with one male and one female used per litter per experimental group. All procedures were approved by New York University and Nathan Kline Institute's Animal Care and Use Committee, in accordance with National Institutes of Health's guidelines for the care and use of laboratory animals.

\section{Procedures}

\section{Scarcity-Adversity Rearing}

On PN8, litters were randomly assigned into scarcity-adversity or control rearing conditions. In scarcity-adversity conditions the mother was provided with insufficient wood shavings materials $(100 \mathrm{ml})$ for nest building in polypropylene cages $(34 \times 29 \times 17 \mathrm{~cm})$, so that she could not build a proper nest for her pups. This procedure has previously been demonstrated to negatively disrupt mother-infant interactions (Perry et al., 2019) and increase pup corticosterone release (Raineki et al., 2010). Scarcity-adversity rearing conditions persisted from PN812. This procedure has been used previously by our lab and others (Roth and Sullivan, 2005; Cui et al., 2006; Raineki et al., 2010, 2012, 2015; Perry and Sullivan, 2014; Rincón-Cortés and Sullivan, 2016; Doherty et al., 2017; Walker et al., 2017).

\section{Peer Housing Intervention}

After weaning at PN23, animals were pair-housed in polypropylene cages $(34 \times 29 \times 17 \mathrm{~cm})$ based on matched or mismatched early-life rearing conditions. In matched housing conditions, two age- and sex- matched control reared rats were housed together, or two age- and sex-matched scarcity-adversity reared rats were housed together. In mismatched housing conditions, one control reared rat and one scarcity-adversity reared rat (age and sex matched) were housed together. For all housing conditions, animals were supplied with ad libitum food (Purina LabDiet \#5001) and water, as well as ample wood shavings materials and a plastic tube. Peer housing conditions were maintained for at least 2 weeks, spanning from weaning at PN23 until time of testing in peri-adolescence (PN37-47).

\section{Spontaneous Alternation Task}

Spatial working memory was assessed using a spontaneous alternation task, which is based on the natural proclivity of rodents to sequentially alternate between arms during exploration of a T- or Y-maze (Lalonde, 2002). Subjects were tested one time only in peri-adolescence (PN37-47) using a Y-maze apparatus $(76.2 \times 64.8 \times 18.1 \mathrm{~cm})$. The apparatus was constructed with a black Plexiglas floor and walls, and a clear Plexiglas lid. The maze did not contain any visual cues, but extra-maze cues were visible from all three arms to allow spatial orientation. The subject was placed in the center of the Y-maze and allowed to freely roam the apparatus for the duration of the 8-min task. All testing occurred during the light period (ZT3-ZT7, zeitgeber time, ZT0 represents light on/ZT12 represents light off). Behavior was recorded using a video camera positioned approximately $1.5 \mathrm{~m}$ above the apparatus. The number and sequence of arm entries were manually scored offline by an observer blinded to experimental 
conditions. Spontaneous alternation consists of sequential entry into each of the three arms. Therefore, percentage of spontaneous alternations was calculated by dividing the total number of alternations by the number of possible alternations: [number of alternations/(number of total arm entries - 2) ]* 100 . Through continuous assessment of spontaneous alternation, this task provides the advantage of allowing the experimenter to avoid repetitive stressful handling of subjects, such as occurs in trial-based assessments of working memory. Furthermore, this spontaneous alternation task allows for the measure of locomotor activity, as indicated by the frequency of arm entries (Hughes, 2004).

\section{Social Behavior Task}

Social behavior was assessed using a two-chamber Plexiglas apparatus $(45.5 \times 30.5 \times 45 \mathrm{~cm})$. The chambers were divided by a Plexiglas division with a square opening $(8 \times 6 \mathrm{~cm})$ that allowed animals to cross between chambers. Two metal cubes $(6 \times 6 \times 6 \mathrm{~cm})$ with $1-\mathrm{cm}$ circular holes were placed in each chamber. The subject was acclimated to the apparatus for $5 \mathrm{~min}$ prior to the start of testing. Animals were excluded from testing if they did not habituate to both chambers (spent less than $20 \%$ of time in either chamber). This exclusion criterion led to the exclusion of one control reared rat (in matched postweaning housing) when tested in peri-adolescence. Following the acclimation period, a younger (PN25-35), same-sex animal was placed inside of the metal cube in the social stimulus chamber, while the metal cube of the other chamber remained empty. The test subject was then placed in the chamber without the social stimulus and allowed to freely roam the apparatus for the duration of the 10-min task. All testing occurred during the light period (ZT3-ZT7, zeitgeber time, ZT0 represents light on/ZT12 represents light off). Testing was recorded using Ethovision software (Noldus, Leesburg, VA, United States). Social behavior was quantified as the total time spent in each chamber, with decreased time spent in the chamber containing the social stimulus relative to the non-social chamber defined as social avoidance (Toth and Neumann, 2013). Number of crossings between chambers was also measured as an index of general locomotor activity (Raineki et al., 2012; Rincón-Cortés and Sullivan, 2016). All behavior was manually scored from videos by an observer blinded to the experimental conditions. Subjects were tested one time only in a social behavior task at either pre-weaning (juvenile; PN20-22) or peri-adolescence (PN37-47), to assess social behavior at ages immediately preceding and following the peer socialization intervention which spanned from PN23 until time of testing in peri-adolescence (PN37-47).

\section{Statistical Analysis}

All experimental data were analyzed using Prism 7 (GraphPad Software, Inc., San Diego, CA) using two-tailed Student's $t$-tests for paired comparisons or two-way ANOVA, followed by post hoc Fisher's LSD tests between groups. Significance of results was accepted at $p<0.05$. Tests were designed assuming normal distribution and variance for control versus scarcity-adversity groups. A priori power analyses using $\mathrm{G}^{*}$ Power 3.1 software indicated that a minimum final group size of six to eight rats was required to have a probability of detecting significant group effects, depending on the experiment. Specifically, power calculation of $t$-tests comparing early-life experience indicated that a minimum sample size of six (Figure 1B) or eight (Figure 3B) rats per group was necessary to achieve power of 0.8 and an error probability of 0.05 . Similar power analysis calculated the requirement of a minimum sample size of six rats per experimental group for two-way ANOVA to achieve power of 0.8 and an error probability of 0.05 (Figures 2B, 3C). All data were checked for statistical outliers using Grubbs' outlier test. One significant outlier was removed from the control reared, mismatched housing condition for Figure 3C. Final sample sizes are as follows: Figure 1B-8 control, 7 scarcityadversity; Figure $2 \mathbf{B}-8$ control matched, 8 scarcity-adversity, 7 control mismatched, 7 scarcity-adversity mismatched; Figure 3B-8 control, 8 scarcity-adversity; Figure 3C-8 control matched, 8 scarcity-adversity matched, 8 control mismatched, 8 scarcity-adversity mismatched.

\section{RESULTS}

Our rodent model of early-life scarcity-adversity exposure significantly reduced peri-adolescent (PN37-47) subjects' spatial working memory as assessed via spontaneous alternations in a Y-maze (Figure 1A), relative to control reared subjects [Figure 1B; $t_{(13)}=3.10, p=0.01$, Cohen's $d=1.602, t$ test]. Sensitivity analyses revealed that group differences in spontaneous alternations were not driven by differences in locomotor activity, as assessed via overall number of arm entries during the task [Figure 1C, $t_{(13)}=1.02, p=0.33$, $t$-test]. Additionally, $t$-tests indicated there were no group differences in percentage of entries into arm A [Figure 1D, $t_{(13)}=0.15$, $p=0.89$ ], arm $\mathrm{B}$ [Figure $1 \mathrm{D}, t_{(13)}=0.25, p=0.81$ ], or arm $\mathrm{C}$ [Figure 1D, $t_{(13)}=0.31, p=0.76$ ] of the maze. Finally, one-sample $t$-tests comparing mean percent arm entries to chance performance ( $33 \%$ entry per arm) indicated that neither experimental group displayed a preference in entering arm A [Figure 1D, Control- $t_{(7)}=0.41, p=0.69$, Scarcity-adversity$\left.t_{(6)}=0.42, p=0.69\right]$, arm B [Figure 1D, Control $-t_{(7)}=0.72$, $p=0.50$, Scarcity-adversity- $\left.t_{(6)}=1.25, p=0.26\right]$, or arm C [Figure 1D, Control- $t_{(7)}=0.50, p=0.63$, Scarcity-adversity$\left.t_{(6)}=0.77, p=0.47\right]$.

However, if scarcity-adversity exposed subjects were housed with a control reared peer from weaning (PN23) until time of testing in peri-adolescence (PN37-47) (Figure 2A), the negative effect of early-life scarcity-adversity rearing on later-life working memory was attenuated. Specifically, results of a $2 \times 2$ ANOVA revealed a significant interaction of early-life experience (scarcity-adversity vs. control) and peer housing condition (matched vs. mismatched) on percentage of spontaneous alternations in a Y-maze [Figure 2B; $F_{(1,26)}=4.67, p=0.04$ ]. Post hoc tests indicated that scarcity-adversity subjects placed in mismatched peer housing conditions were significantly improved in percentage of spontaneous alternations relative to scarcity-adversity subjects in matched peer housing conditions $(p<0.05$, Cohen's $d=0.85)$. Furthermore, scarcity-adversity subjects placed in mismatched peer housing did not significantly 
A

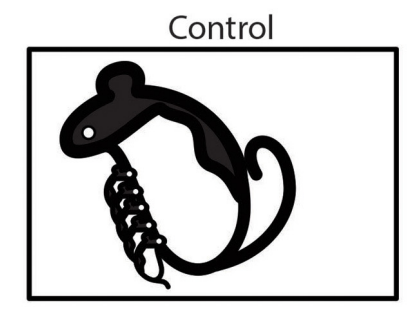

Scarcity-Adversity
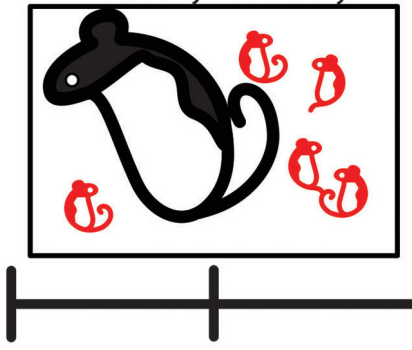

INFANCY

(PN8-12)
Working Memory

B

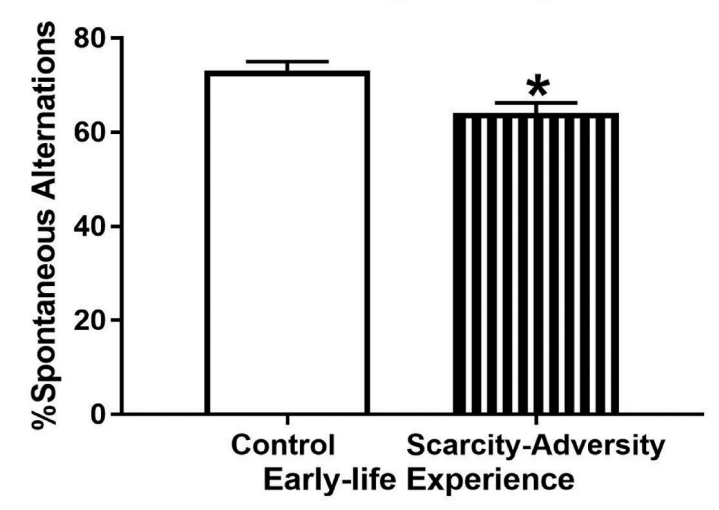

C Arm Entries

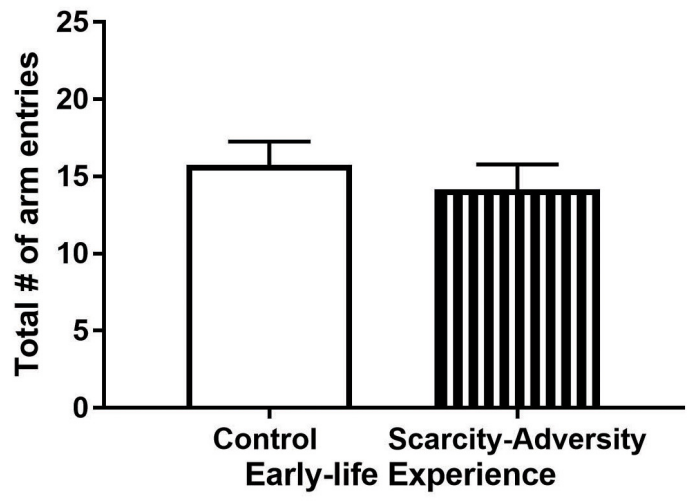

D

$\%$ Entries to Arm

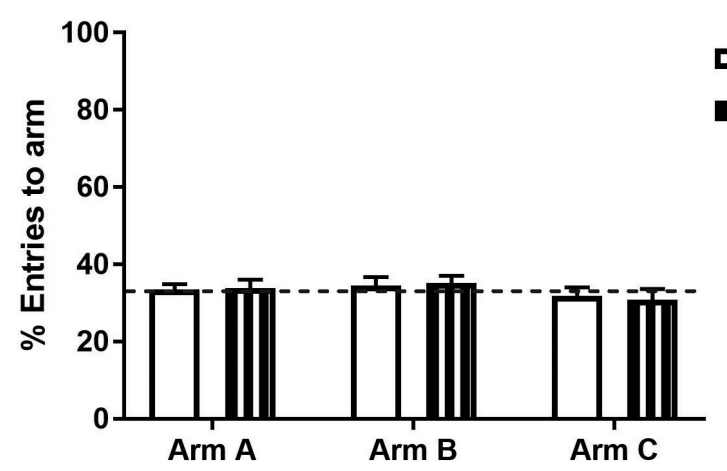

FIGURE 1 | Early-life scarcity-adversity rearing reduced spatial working memory in peri-adolescence. (A) Experimental timeline. (B) Mean ( \pm SEM) levels of percent spontaneous alternation in the spontaneous alternation task (*significant difference between groups, $p<0.05, n=7-8 /$ group). (C) Mean ( \pm SEM) levels of total number of maze arm entries during spontaneous alternation task ( $n=7-8 /$ group). (D) Mean ( \pm SEM) percent levels of entries into each individual arm of the Y-maze during the spontaneous alternation task (dotted line represents level of entries at chance, i.e., $33 \% ; n=7-8 /$ group). 
A

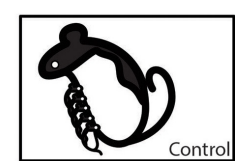

(6)

Control
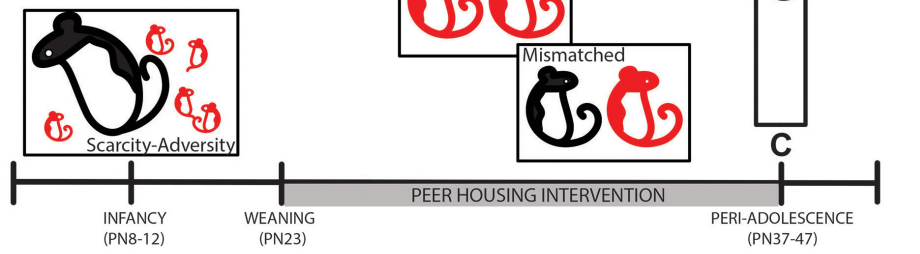

口 Control

m Scarcity-Adversity

\section{8}

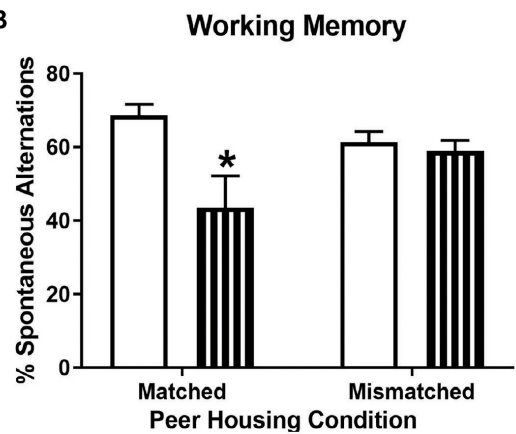

D

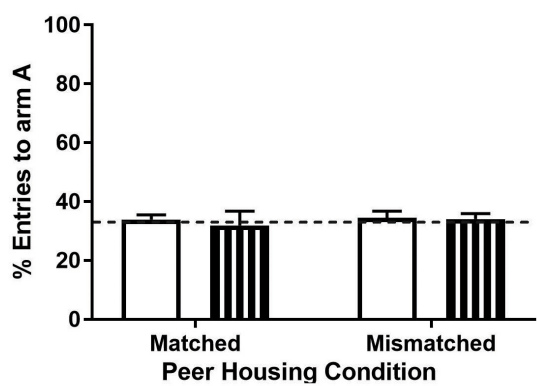

C

Arm Entries

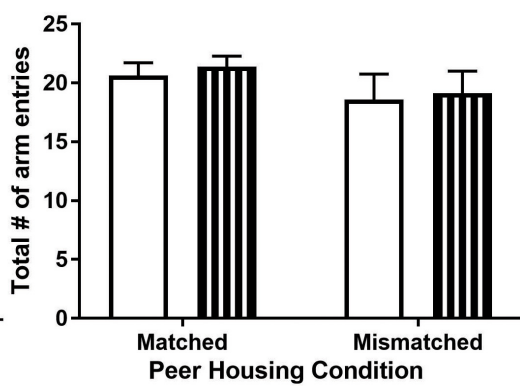

E

$\%$ Entries to Arm B

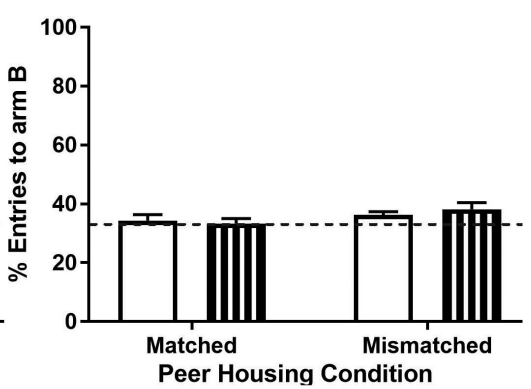

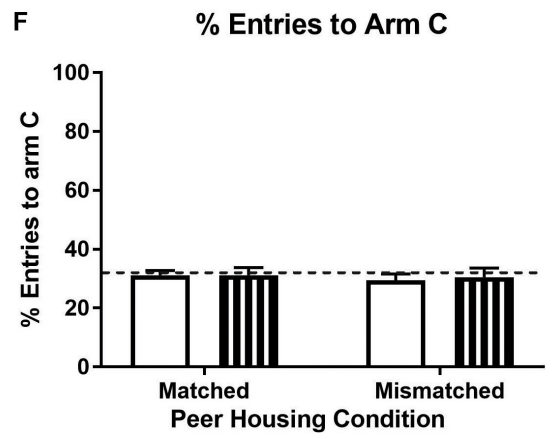

FIGURE 2 | A peer housing intervention rescued spatial working memory following early-life scarcity-adversity exposure. (A) Experimental timeline. (B) Mean ( \pm SEM) levels of percent spontaneous alternation in the spontaneous alternation task (*significantly different from all groups, $p<0.05, n=7-8 / g r o u p)$. (C) Mean ( \pm SEM) levels of total number of maze arm entries during spontaneous alternation task ( $n=7-8 /$ group). (D) Mean ( \pm SEM) percent levels of entries into arm A of the Y-maze during the spontaneous alternation task (dotted line represents level of entries at 33\%; $n=7-8 /$ group). (E) Mean ( \pm SEM) percent levels of entries into arm B of the Y-maze during the spontaneous alternation task (dotted line represents level of entries at 33\%; $n=7-8 /$ group). (F) Mean ( \pm SEM) percent levels of entries into arm $\mathrm{C}$ of the $\mathrm{Y}$-maze during the spontaneous alternation task (dotted line represents level of entries at chance, i.e., 33\%; $n=7-8 /$ group). 
A

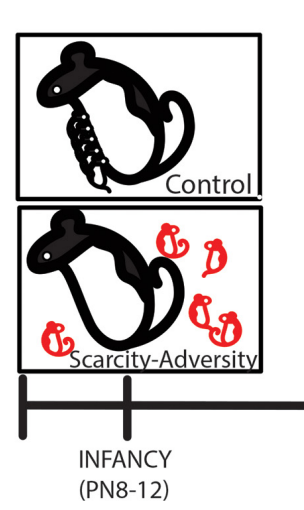

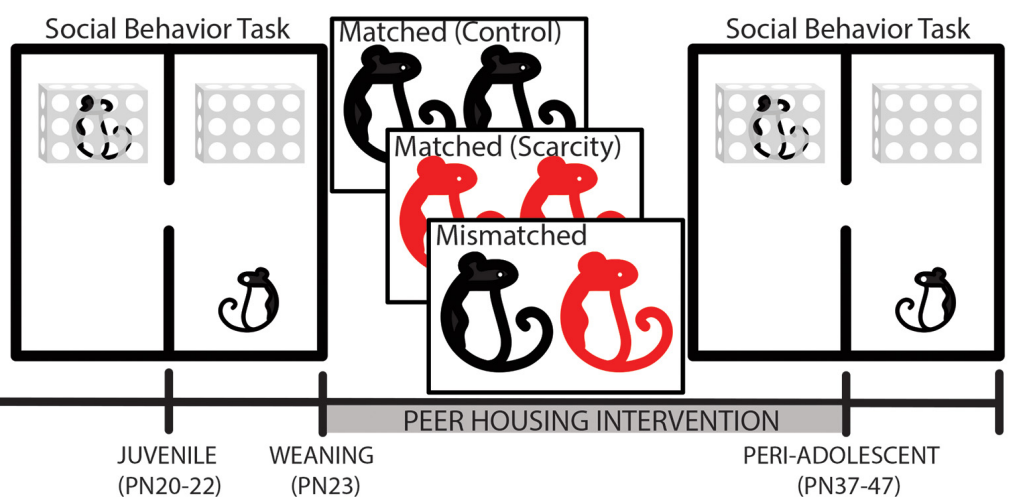

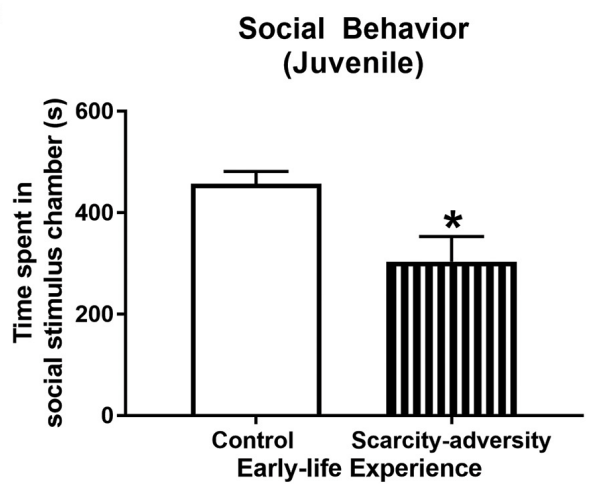

C

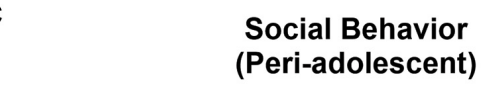

FIGURE 3 | Scarcity-adversity reared subjects had improved social behavior following the peer housing intervention. (A) Experimental timeline. (B) Mean ( \pm SEM) time spent in the social stimulus chamber during the social behavior test (*significant difference between groups, $p<0.05, n=8 /$ group). (C) Mean ( \pm SEM) time spent in the social stimulus chamber during the social behavior test (*significant difference between groups, $p<0.05, n=8 /$ group).

differ from control reared subjects in their levels of spontaneous alternation (post hoc tests, $p<0.05$ ). Sensitivity analyses indicated that group differences in spontaneous alternations were not driven by differences in overall number of arm entries during the task [Figure 2C, interaction $-F_{(1,26)}=0.01, p=0.95$; main effect of peer housing $-F_{(1,26)}=1.94, p=0.18$, main effect of early-life experience- $F_{(1,26)}=0.18, p=0.67,2 \times 2$ ANOVA]. Furthermore, $2 \times 2$ ANOVAs revealed that there were no group differences in percentage of entries to arm A [Figure 2D, interaction $-F_{(1,26)}=0.07, p=0.79$; main effect of peer housing $-F_{(1,26)}=0.20, p=0.66$, main effect of early-life experience- $F_{(1,26)}=0.15, p=0.70,2 \times 2$ ANOVA], arm B [Figure 2E, interaction $-F_{(1,26)}=0.63, p=0.43$; main effect of peer housing $-F_{(1,26)}=3.38, p=0.08$, main effect of early-life experience- $F_{(1,26)}=0.07, p=0.79,2 \times 2$ ANOVA], or arm C [Figure 2F, interaction $-F_{(1,26)}=0.05, p=0.83$; main effect of peer housing $-F_{(1,26)}=0.24, p=0.63$, main effect of early-life experience- $F_{(1,26)}=0.04, p=0.84,2 \times 2$ ANOVA] of the maze. Lastly, one-sample $t$-tests comparing mean percent arm entries to chance performance (33\% entry per arm) indicated that neither experimental group displayed a preference in entering arm A [Figure 2D, Control Matched- $t_{(7)}=0.59$, $p=0.57$, Scarcity-adversity Matched $-t_{(7)}=0.21, p=0.84$, Control Mismatched- $t_{(6)}=0.66, p=0.53$, Scarcity-adversity Mismatched $-t_{(6)}=0.64, p=0.55$ ], arm B [Figure 2E, Control
Matched $-t_{(7)}=0.67, p=0.52$, Scarcity-adversity Matched$t_{(7)}=0.25, p=0.81$, Control Mismatched $-t_{(6)}=0.17$, $p=0.87$, Scarcity-adversity Mismatched-t $t_{(6)}=0.1 .45$, $p=0.20$ ], or arm C [Figure 2F, Control Matched $-t_{(7)}=1.12$, $p=0.30$, Scarcity-adversity Matched $-t_{(7)}=0.69, p=0.51$, Control Mismatched $-t_{(6)}=1.68, p=0.15$, Scarcity-adversity Mismatched $\left.-t_{(6)}=0.79, p=0.46\right]$.

Lastly, we checked if our peer housing mismatched condition improved scarcity-adversity reared subjects' social behavior, as intended (Figure 3A). Pre-weaning juvenile (PN20-22) scarcityadversity reared subjects displayed a significant reduction in time spent with a social stimulus rat during the social behavior task relative to control reared subjects [Figure $3 \mathbf{B} ; t_{(14)}=2.78$, $p=0.015$, Cohen's $d=1.39$, $t$-test]. Furthermore, assessment of social behavior in peri-adolescence (following the peer housing intervention) revealed a significant interaction of early-life experience and post-weaning housing condition on time spent with a social stimulus [Figure 3C; $F_{(1,28)}=9.49, p=0.01$, $2 \times 2$ ANOVA]. Specifically, if scarcity-adversity reared subjects were housed in matched peer housing conditions, they displayed reduced time spent with a social stimulus rat relative to control reared subjects (post hoc tests, $p<0.05$, Cohen's $d=1.53$ ). However, if scarcity-adversity reared subjects were housed in mismatched peer housing conditions, they did not differ from control subjects in time spent with a social stimulus rat (post hoc 
tests, $p<0.05$ ), and spent significantly more time with a social stimulus relative to scarcity-adversity subjects in matched peer housing conditions (post hoc tests, $p<0.05$, Cohen's $d=1.52$ ).

\section{DISCUSSION}

It has long been theorized that humans develop higher mental functions, such as EFs, within the context of interpersonal interactions and social relationships (Vygotsky, 1978; Carlson, 2009; Lewis and Carpendale, 2009; Moriguchi, 2014; van Lier and Deater-Deckard, 2016). In the present study, we began to test the causal relations between social and EF development by using a rodent model to experimentally examine the contributions of peer socialization (the mismatched housing condition) to the development of working memory. Given the lack of research examining social contributions to EF development beyond early childhood, we focused our assessment on the contributions of post-weaning peer socialization on subsequent working memory performance. Specifically, we demonstrated that earlylife scarcity-adversity, as modeled by rearing infant rat pups and their mother with insufficient wood shavings materials for nest building, reduced spatial working memory in periadolescence, as evidenced by reduced spontaneous alternation between arms of Y-maze. Notably, early-life scarcity-adversity did not produce alterations in overall number of arm entries during the Y-maze task, nor did it lead to a preference for entering a specific arm of the maze. Thus, it appears that early-life scarcity-adversity uniquely impacted spontaneous alternation between the maze arms, which we interpret as decreased working memory ability. However, we also found causal evidence that housing a scarcity-adversity reared rat with a control reared rat normalized working memory performance of scarcity-adversity reared peri-adolescents. This mismatched co-housing condition appears to have operated, at least in part, by improving scarcityadversity reared subjects' social behavior, which is consistent with a broad literature supporting that EFs (such as working memory) develop through social interactions and the attainment of appropriate social skills (Vygotsky, 1978; Carlson, 2009; Lewis and Carpendale, 2009; Moriguchi, 2014; van Lier and DeaterDeckard, 2016; Perry et al., 2018b).

Prior research from Sullivan and colleagues established that our early-life scarcity-adversity model induces social avoidance in juvenile, adolescent, and adult rats (Raineki et al., 2012, 2015; Rincón-Cortés and Sullivan, 2016). Here, we replicated and expanded upon these findings by providing novel evidence that this socially avoidant phenotype co-occurs with working memory problems in peri-adolescent rats. Thus, the present study's findings supported our hypothesis that scarcity-adversity rearing would produce cognitive development problems, as evidenced via spatial working memory performance in a Y-maze. Our findings that social behavior and cognitive problems cooccurred by peri-adolescence align with increasing evidence that social and cognitive aspects of development are functionally and reciprocally linked (Riggs et al., 2006; Carlson, 2009; Lewis and Carpendale, 2009; Moriguchi, 2014; van Lier and DeaterDeckard, 2016; Perry et al., 2018b). Furthermore, our findings of scarcity-adversity induced working memory problems are consistent with human literature suggesting that poverty-related adversity negatively impacts EF development (Raver et al., 2013; Ursache et al., 2015; Perry et al., 2018b). Thus, our rodent model of scarcity-adversity appears to be somewhat translationally valid and can be further leveraged to discern behavioral and neurobiological mechanisms by which scarcityadversity exposure influences EF development.

The present study's findings also support our hypothesis that socializing a scarcity-adversity reared subject with a control reared rat (via co-housing) would improve the scarcityadversity reared subject's social behavior and working memory. Specifically, we demonstrated that pair housing a scarcityadversity reared rat with a control reared rat rescued their socially avoidant behavior, as well as spatial working memory in a Y-maze. It is important to note that in our mismatched housing condition, the scarcity-adversity rat was not detrimental to control subjects' social behavior or working memory postintervention. Altogether, these findings are consistent with ours and others' prior human research findings that support a theory of change whereby EFs of at-risk children can be improved by peer-based socialization that promotes the attainment of appropriate social skills. In humans, peers are powerful mediators of learning and gain increasing influence across development (Harris, 1995; Steinberg and Monahan, 2007; Rubin et al., 2011; Cappella et al., 2013; Telzer et al., 2018). Thus, peer-based interventions, particularly in middle childhood and beyond when peers become more central in youth's lives, are of high potential merit for the improvement of child EF outcomes. In human research, individual peer-based socialization interventions have been successfully employed in school-based settings for the improvement of prosocial behavior (Zhang and Wheeler, 2011), externalizing or internalizing problems (Fantuzzo et al., 2005), and learning outcomes (Odom and Strain, 1984; Topping, 1996; Fuchs et al., 2008, 2009). Furthermore, peer-based interventions have leveraged natural opportunities for peer interactions in school settings to successfully overcome high student-to-staff ratios and teacher burden (Bouffard and Little, 2003; Fantuzzo et al., 2005). However, few studies have begun to assess the efficacy of peer-based interventions in improving EFs (Christ et al., 2017).

\section{Limitations and Future Directions}

A major strength of this study was the use of an experimental design that provides high internal validity, allowing for a clearer definition of cause-effect relationships between social experiences and working memory performance. However, the current findings should be interpreted with the following limitations in mind. First and foremost, the high internal validity of the present study's rodent experimental design comes with a trade-off to the study design's external validity. Rodent models cannot encompass the complexity of human conditions (such as social and cultural phenomena), and thus appropriate caution should be taken when interpreting the present study's results (Perry et al., 2019). Additionally, while the present study's rodent findings provide causal support for the notion that peer interactions can be leveraged for the improvement of working 
memory, we have assessed working memory via only one outcome measure. Expanding ways in which working memory (and other measures of EFs) is assessed in rodent experimental designs would strengthen the present study's findings and interpretations.

Indeed, future rodent research should replicate and expand upon the assessment of working memory by exploring if early adversity similarly impacts in other domains of EF development (e.g., cognitive flexibility, inhibitory control). While considered functionally distinct "core" domains of EF, working memory, cognitive flexibility, and inhibitory control are related and typically operate together (Miyake et al., 2000; Friedman and Miyake, 2017). For example, working memory and inhibitory control largely support one another such that one skill is rarely called upon without the other (Diamond, 2013). Furthermore, cognitive flexibility, which develops later, builds upon working memory and inhibitory control (Diamond, 2013). Thus, it is plausible that scarcity-adversity induced differences in working memory might co-occur with problems related to inhibitory control and cognitive flexibility. However, working memory, inhibitory control, and cognitive flexibility differ in their developmental trajectories, and are subserved by overlapping but unique neural networks (for review see Perry et al., 2018a) which could be differentially impacted by scarcityadversity. Thus, it is also plausible that scarcity-adversity might uniquely impact the development of each core EF based on the developmental timing of adversity exposure and/or the mechanisms by which adversity influences the developing brain areas underlying EF development.

The high internal validity of our rodent model also warrants future rodent research to disentangle the mechanisms mediating the functional interplay between social processes and EF development. Indeed, future experiments should attempt to discern the specific mechanisms by which mismatched peer housing conditions improve working memory performance. For example, observations of naturalistic rodent behaviors in the mismatched housing conditions will provide evidence to if and how control reared subjects scaffold scarcityadversity reared subjects' social behavior. It is also possible that benefits of mismatched housing conditions are imparted via less directly observable mechanisms. For example, prior research has identified that microbial reconstitution rescues social behavior deficits in a mouse model of autism spectrum disorder (Buffington et al., 2016). Specifically, Buffington et al. (2016) utilized a mismatched housing intervention whereby offspring of mothers on a high-fat diet (MHFD) were cohoused with offspring of mothers on a regular diet (MRD). These mismatched housing conditions rescued social behavior deficits of MHFD offspring via a mechanism dependent on gut microbiota transfer from MRD offspring to MHFD offspring. Given the impact of gut microbiota on the brain (Cryan and Dinan, 2012), microbial transfer could underlie recovery of both social behavior and working memory. Future experimentation will help discern if similar mechanisms underlie our mismatched housing intervention, and thus provide important insight into means by which to improve EF development. Our rodent model can also be leveraged to determine how benefits to EF outcomes vary as a function of the developmental timing of our mismatched peer intervention, which would provide important insight for peer-based intervention efforts.

\section{CONCLUSION}

In conclusion, the present study has provided novel, causal evidence that a peer-based intervention spanning from immediately post-weaning to peri-adolescence rescues early-life scarcity-adversity induced working memory problems in rodents. Furthermore, the positive effects of this peer-based intervention appear to be operating, at least in part, via the improvement of scarcity-adversity reared subjects' social behavior. These findings converge with our lab's previous human research, as well as prior literature supporting an overarching theory that humans develop higher mental functions such as EFs within the context of interpersonal interactions and social relationships.

To the best of our knowledge, the present findings are the first of its kind using a rodent model, which opens opportunities for studies to assess the specific behavioral and neurobiological mechanisms by which social interactions influence cognitive development. Animal models, when carefully designed and considered within the context of human research findings, provide powerful means for the efficient assessment of theorybased mechanisms of change. Furthermore, animal models have a high potential to contribute to the development of mechanismbased, biologically sensitive interventions. While EF training can be effective in many forms, interventions targeting the improvement of social skills and social interactions may prove to be particularly efficacious and generalizable across context and areas of functioning, and thus should be the focus of continued research.

\section{DATA AVAILABILITY STATEMENT}

The datasets generated for this study are available on request to the corresponding author.

\section{ETHICS STATEMENT}

The animal study was reviewed and approved by New York University and Nathan Kline Institute's Animal Care and Use Committee.

\section{AUTHOR CONTRIBUTIONS}

$\mathrm{RP}, \mathrm{RS}, \mathrm{MO}, \mathrm{CA}$, and $\mathrm{CB}$ contributed to the conception and design of the study. RP performed rodent experiments and statistical analyses, and wrote the first draft of the manuscript. SB, MR-C, and DC performed rodent experiments. DC created illustrations for the figures. AB-A helped write sections of the manuscript. All authors contributed to manuscript revision, and read and approved the submitted version. 


\section{FUNDING}

This material is based on work supported by the National Science Foundation under Grant No. 1810208 awarded to RP. SB and $\mathrm{AB}-\mathrm{A}$ were supported by the National Science Foundation Graduate Research Fellowship Program, under Grant No.

\section{REFERENCES}

Bateson, P. (2005). The Role of Play in the Evolution Of Great Apes and Humans. New York, NY: Guilford Press.

Baumeister, R. F., DeWall, C. N., Ciarocco, N. J., and Twenge, J. M. (2005). Social exclusion impairs self-regulation. J. Pers. Soc. Psychol. 88:589. doi: 10.1037/ 0022-3514.88.4.589

Baumeister, R. F., Twenge, J. M., and Nuss, C. K. (2002). Effects of social exclusion on cognitive processes: anticipated aloneness reduces intelligent thought. J. Pers. Soc. Psychol. 83:817. doi: 10.1037/0022-3514.83.4.817

Bibok, M. B., Carpendale, J. I., and Muller, U. (2009). Parental scaffolding and the development of executive function. New Dir. Child Adolesc. Dev. 2009, 17-34. doi: $10.1002 / \mathrm{cd} .233$

Blair, C., Kuzawa, C. W., and Willoughby, M. T. (2019). The development of executive function in early childhood is inversely related to change in body mass index: evidence for an energetic trade-off? Dev. Sci. 18:e12860. doi: 10. $1111 /$ desc. 12860

Blair, C., and Raver, C. C. (2014). Closing the achievement gap through modification of neurocognitive and neuroendocrine function: results from a cluster randomized controlled trial of an innovative approach to the education of children in Kindergarten. PLoS One 9:e112393. doi: 10.1371/journal.pone. 0112393

Bodrova, E., and Leong, D. J. (1996). Tools of the Mind: The Vygotskian Approach to Early Childhood Education. Englewood Cliffs, NJ: Merrill, an imprint of Prentice Hall.

Bouffard, S., and Little, P. M. D. (2003). A review of Activity Implementation in Out-of-School Time Programs. Cambridge, MA: Harvard Family Research Project.

Buffington, S. A., Di Prisco, G. V., Auchtung, T. A., Ajami, N. J., Petrosino, J. F., and Costa-Mattioli, M. (2016). Microbial reconstitution reverses maternal dietinduced social and synaptic deficits in offspring. Cell 165, 1762-1775. doi: 10.1016/j.cell.2016.06.001

Cappella, E., Kim, H. Y., Neal, J. W., and Jackson, D. R. (2013). Classroom peer relationships and behavioral engagement in elementary school: the role of social network equity. Am. J. Commun. Psychol. 52, 367-379. doi: 10.1007/s10464013-9603-5

Carlson, S. M. (2009). Social origins of executive function development. New Dir. Child Adolesc. Dev. 2009, 87-98. doi: 10.1002/cd.237

Christ, S. E., Stichter, J. P., O'Connor, K. V., Bodner, K., Moffitt, A. J., and Herzog, M. J. (2017). Social skills intervention participation and associated improvements in executive function performance. Autism Res. Treatment. 2017:5843851. doi: 10.1155/2017/5843851

Cryan, J. F., and Dinan, T. G. (2012). Mind-altering microorganisms: the impact of the gut microbiota on brain and behaviour. Nat. Rev. Neurosci. 13, 701-712. doi: $10.1038 / \mathrm{nrn} 3346$

Cui, M., Yang, Y., Yang, J., Zhang, J., Han, H., Ma, W., et al. (2006). Enriched environment experience overcomes the memory deficits and depressive-like behavior induced by early life stress. Neurosci. Lett. 404, 208-212. doi: 10.1016/ j.neulet.2006.05.048

Diamond, A. (2013). Executive functions. Annu. Rev. Psychol. 64, 135-168. doi: 10.1146/annurev-psych-113011-143750

Diamond, A., Barnett, W. S., Thomas, J., and Munro, S. (2007). Preschool program improves cognitive control. Science 318, 1387-1388. doi: 10.1126/ science. 1151148

Doherty, T. S., Blaze, J., Keller, S. M., and Roth, T. L. (2017). Phenotypic outcomes in adolescence and adulthood in the scarcity-adversity model of low nesting resources outside the home cage. Dev. Psychobiol. 59, 703-714. doi: 10.1002/ dev. 21547
DGE1342536. Part of the work described herein was also supported by the Eunice Kennedy Shriver National Institute of Child Health and Human Development (R37-HD083217 to RS). Any opinions, findings, and conclusions or recommendations expressed in this material are those of the authors and do not necessarily reflect the views of the National Science Foundation.

Fantuzzo, J., Manz, P., Atkins, M., and Meyers, R. (2005). Peer-mediated treatment of socially withdrawn maltreated preschool children: cultivating natural community resources. J. Clin. Child Adolesc. Psychol. 34, 320-325. doi: 10.1207/s15374424jccp3402_11

Friedman, N. P., and Miyake, A. (2017). Unity and diversity of executive functions: individual differences as a window on cognitive structure. Cortex 86, 186-204. doi: 10.1016/j.cortex.2016.04.023

Fuchs, D., Fuchs, L. S., Simmons, D. C., and Mathes, P. G. (2008). Peer-Assisted Learning Strategies: Reading Methods for Grades 2-6, 2008. Nashville, TN: Vanderbilt University.

Fuchs, L. S., Fuchs, D., Karns, K., and Phillips, N. B. (2009). Peer-Assisted Learning Strategies: Math Methods for Grades 2-6 Teacher Manual, 2009. Nashville, TN: Vanderbilt University.

Fuhrmann, D., Knoll, L. J., and Blakemore, S. J. (2015). Adolescence as a sensitive period of brain development. Trends in Cogn. Sci. 19, 558-566. doi: 10.1016/j. tics.2015.07.008

Harris, J. R. (1995). Where is the child's environment? a group socialization theory of development. Psychol. Rev. 102, 458-489. doi: 10.1037/0033-295X.102.3.458

Holmes, C. J., Kim-Spoon, J., and Deater-Deckard, K. (2016). Linking executive function and peer problems from early childhood through middle adolescence. J. Abnorm. Child Psychol. 44, 31-42. doi: 10.1007/s10802-015-0044-5

Hughes, C. H., and Ensor, R. A. (2009). How do families help or hinder the emergence of early executive function? New Direct. Child Adolesc. Dev. 2009, 35-50. doi: 10.1002/cd.234

Hughes, R. N. (2004). The value of spontaneous alternation behavior (SAB) as a test of retention in pharmacological investigations of memory. Neurosci. Biobehav. Rev. 28, 497-505. doi: 10.1016/j.neubiorev.2004.06.006

Klingberg, T., Fernell, E., Olesen, P. J., Johnson, M., Gustafsson, P., Dahlström, K., et al. (2005). Computerized training of working memory in children with ADHD-A randomized, controlled trial. J. Am. Acad. Child Adolesc. Psychiatr. 44, 177-186. doi: 10.1097/00004583-200502000-200502010

Klingberg, T., Forssberg, H., and Westerberg, H. (2002). Training of working memory in children with ADHD. J. Clin. Exp. Neuropsychol. 24, 781-791. doi: 10.1076/jcen.24.6.781.8395

Kraeuter, A. K., Guest, P. C., and Sarnyai, Z. (2019). The Y-Maze for Assessment of Spatial Working and Reference Memory in Mice. In Pre-Clinical Models. New York, NY: Humana Press.

Lalonde, R. (2002). The neurobiological basis of spontaneous alternation. Neurosci. Biobehav. Rev. 26, 91-104. doi: 10.1016/S0149-7634(01)00041-0

Landry, S. H., Miller-Loncar, C. L., Smith, K. E., and Swank, P. R. (2002). The role of early parenting in children's development of executive processes. Dev. Neuropsychol. 21, 15-41. doi: 10.1207/S15326942DN2101-2

Lewis, C., and Carpendale, J. I. (2009). Introduction: links between social interaction and executive function. New Dir. Child Adolesc. Dev. 2009, 1-15. doi: $10.1002 / \mathrm{cd} .232$

Liet, C., Amenouche, F., Freret, T., Boulouard, M., Mauvieux, B., LelongBoulouard, V., et al. (2015). Effects of acute administration of melatonin on attentional, executive, and working memory processes in rats. Fundamental Clin. Pharmacol. 29, 472-477. doi: 10.1111/fcp.12134

Lindsey, E. W., and Colwell, M. J. (2003). Preschoolers' emotional competence: links to pretend and physical play. Child Stud. J. 33, 39-53.

Miyake, A., Friedman, N. P., Emerson, M. J., Witzki, A. H., Howerter, A., and Wager, T. D. (2000). The unity and diversity of executive functions and their contributions to complex "Frontal Lobe" tasks: a latent variable analysis. Cogn. Psychol. 41, 49-100. doi: 10.1006/cogp.1999.0734

Moriguchi, Y. (2014). The early development of executive function and its relation to social interaction: a brief review. Front. Psychol. 5:388. doi: 10.3389/fpsyg. 2014.00388 
Odom, S. L., and Strain, P. S. (1984). Peer-mediated approaches to promoting children's social interaction: a review. Am. J. Orthopsychiatr. 54:544. doi: 10. 1111/j.1939-0025.1984.tb01525.x

Perry, R., and Sullivan, R. M. (2014). Neurobiology of attachment to an abusive caregiver: short-term benefits and long-term costs. Dev. Psychobiol. 56, 16261634. doi: 10.1002/dev.21219

Perry, R. E., Braren, S. H., Blair, C., and The Family Life Project Key Investigators, (2018a). Socioeconomic risk and school readiness: longitudinal mediation through children's social competence and executive function. Front. Psychol. 9:1544. doi: 10.3389/fpsyg.2018.01544

Perry, R. E., Finegood, E. D., Braren, S. H., and Blair, C. (2018b). “The social neuroendocrinology and development of executive functions," in Routledge International Handbook of Social Neuroendocrinology, eds O. C. Schultheiss, and P. H. Mehta, (London: Routledge), 530-543. doi: 10.4324/97813152004 39-30

Perry, R. E., Finegood, E. D., Braren, S. H., DeJoseph, M. L., Putrino, D. F., Wilson, D. A., et al. (2019). Developing a neurobehavioral animal model of poverty: drawing cross-species connections between environments of scarcity-adversity, parenting quality, and infant outcome. Dev. Psychopathol. 31, 399-418. doi: 10.1017/S095457941800007X

Peterson, J. B., and Flanders, J. L. (2005). Play and the Regulation of Aggression. New York, NY: Guilford Press.

Raineki, C., Moriceau, S., and Sullivan, R. M. (2010). Developing a neurobehavioral animal model of infant attachment to an abusive caregiver. Biol. Psychiatry 67, 1137-1145. doi: 10.1016/j.biopsych.2009.12.019

Raineki, C., Rincón-Cortés, M. R., Belnoue, L., and Sullivan, R. M. (2012). Effects of early-life abuse differ across development: infant social behavior deficits are followed by adolescent depressive-like behaviors mediated by the amygdala. J. Neurosci. 32, 7758-7765. doi: 10.1523/JNEUROSCI.5843-11. 2012

Raineki, C., Sarro, E., Rincón-Cortés, M., Perry, R., Boggs, J., Holman, C. J., et al. (2015). Paradoxical neurobehavioral rescue by memories of early-life abuse: the safety signal value of odors learned during abusive attachment. Neuropsychopharmacology 40, 906-914. doi: 10.1038/npp.2014.266

Raver, C. C., Blair, C., and Willoughby, M. (2013). Poverty as a predictor of 4-year-olds' executive function: new perspectives on models of differential susceptibility. Dev. Psychol. 49:292. doi: 10.1037/a0028343

Riggs, N. R., Jahromi, L. B., Razza, R. P., Dillworth-Bart, J. E., and Mueller, U. (2006). Executive function and the promotion of social-emotional competence. J. Appl. Dev. Psychol. 27, 300-309. doi: 10.1016/j.appdev.2006. 04.002

Rincón-Cortés, M., and Sullivan, R. M. (2016). Emergence of social behavior deficit, blunted corticolimbic activity and adult depression-like behavior in a rodent model of maternal maltreatment. Transl. Psychiatry 6:e930. doi: 10.1038/tp. 2016.205

Roskam, I., Stievenart, M., Meunier, J. C., and Noël, M. P. (2014). The development of children's inhibition: does parenting matter? J. Exp. Child Psychol. 122, 166-182. doi: 10.1016/j.jecp.2014.01.003

Roth, T. L., and Sullivan, R. M. (2005). Memory of early maltreatment: neonatal behavioral and neural correlates of maternal maltreatment within the context of classical conditioning. Biol. Psychiatry 57, 823-831. doi: 10.1016/j.biopsych. 2005.01.032

Rubin, K. H., Bukowski, W. M., and Laursen, B. (eds) (2011). Handbook of Peer Interactions, Relationships, and Groups. New York, NY: Guilford Press.

Sasser, T. R., Bierman, K. L., Heinrichs, B., and Nix, R. L. (2017). Preschool intervention can promote sustained growth in the executive-function skills of children exhibiting early deficits. Psychol. Sci. 28, 1719-1730. doi: 10.1177/ 0956797617711640
Schulz, K. M., Zehr, J. L., Salas-Ramirez, K. Y., and Sisk, C. L. (2009). Testosterone programs adult social behavior before and during, but not after, adolescence. Endocrinology 150, 3690-3698. doi: 10.1210/en.2008-1708

Sisk, C. L., and Foster, D. L. (2004). The neural basis of puberty and adolescence. Nat. Neurosci. 7:1040. doi: 10.1038/nn1326

Steinberg, L., and Monahan, K. C. (2007). Age differences in resistance to peer influence. Dev. Psychol. 43:1531. doi: 10.1037/0012-1649.43.6.1531

Telzer, E. H., Van Hoorn, J., Rogers, C. R., and Do, K. T. (2018). Social influence on positive youth development: a developmental neuroscience perspective. $A d v$. Child Dev. Behav. 54, 215-258. doi: 10.1016/bs.acdb.2017.10.003

Topping, K. J. (1996). The effectiveness of peer tutoring in further and higher education: a typology and review of the literature. High. Educ. 32, 321-345. doi: $10.1007 /$ BF00138870

Toth, I., and Neumann, I. D. (2013). Animal models of social avoidance and social fear. Cell Tissue Res. 354, 107-118. doi: 10.1007/s00441-013-1636-4

Ursache, A., Noble, K. G., and Blair, C. (2015). Socioeconomic status, subjective social status, and perceived stress: associations with stress physiology and executive functioning. Behav. Med. 41, 145-154. doi: 10.1080/08964289.2015. 1024604

van Lier, P. A., and Deater-Deckard, K. (2016). Children's elementary school social experience and executive functions development: introduction to a special section. J. Abnorm. Child Psychol. 44, 1-6. doi: 10.1007/s10802-015-0113-9

Vecera, S. P., Rothbart, M. K., and Posner, M. I. (1991). Development of spontaneous alternation in infancy. J. Cogn. Neurosci. 3, 351-354. doi: 10.1162/ jocn.1991.3.4.351

Verburgh, L., Königs, M., Scherder, E. J., and Oosterlaan, J. (2014). Physical exercise and executive functions in preadolescent children, adolescents and young adults: a meta-analysis. Br. J. Sports Med. 48, 973-979. doi: 10.1136/ bjsports-2012-091441

Vygotsky, L. S. (1978). Mind in Society: The Development of Higher Psychological Processes. Cambridge: Harvard University Press.

Walker, C. D., Bath, K. G., Joels, M., Korosi, A., Larauche, M., Lucassen, P. J., et al. (2017). Chronic early life stress induced by limited bedding and nesting (LBN) material in rodents: critical considerations of methodology, outcomes and translational potential. Stress 20,421-448. doi: 10.1080/10253890.2017.134 3296

Wei, L., Meaney, M. J., Duman, R. S., and Kaffman, A. (2011). Affiliative behavior requires juvenile, but not adult neurogenesis. J. Neurosci. 31, 14335-14345. doi: 10.1523/JNEUROSCI.1333-11.2011

Zelazo, P. D., and Lyons, K. E. (2012). The potential benefits of mindfulness training in early childhood: a developmental social cognitive neuroscience perspective. Child Dev. Perspect. 6, 154-160. doi: 10.1111/j.1750-8606.2012. 00241.x

Zhang, J., and Wheeler, J. J. (2011). A meta-analysis of peer-mediated interventions for young children with autism spectrum disorders. Educ. Train. Autism Dev. Disabil. 46, 62-77.

Conflict of Interest: The authors declare that the research was conducted in the absence of any commercial or financial relationships that could be construed as a potential conflict of interest.

Copyright (c) 2019 Perry, Braren, Rincón-Cortés, Brandes-Aitken, Chopra, Opendak, Alberini, Sullivan and Blair. This is an open-access article distributed under the terms of the Creative Commons Attribution License (CC BY). The use, distribution or reproduction in other forums is permitted, provided the original author(s) and the copyright owner(s) are credited and that the original publication in this journal is cited, in accordance with accepted academic practice. No use, distribution or reproduction is permitted which does not comply with these terms. 